\title{
Spinal Cord Stimulation for Chronic Refractory Neuropathic Pain: A Technical Note Initial Experience of Two cases
}

\author{
Sanjeev Srivastava ${ }^{1, \odot} \quad$ Pawan Goyal $^{1} \quad$ Anurag Sharma ${ }^{1, \odot}$ Sanjay K. Rajan ${ }^{1} \quad$ Aditya Gupta $^{1}$ \\ ${ }^{1}$ Artemis Agrim Institute of Neurosciences, Artemis Hospital, \\ Gurgaon, Haryana, India \\ Address for correspondence Sanjeev Srivastava, MBBS, DNB, \\ Artemis Agrim Institute of Neurosciences, Artemis Hospital, \\ Gurgaon 122001, Haryana, India \\ (e-mail: sanjeev.srivastava@gmail.com).
}

Indian J Neurosurg 2021;10:244-252.

\begin{abstract}
Spinal cord stimulation is an established procedure for relieving chronic neuropathic pain conditions. Although it has been over five decades since the first spinal cord stimulation (SCS) was developed, it has only been used in a few cases in India. It is primarily based on the "Gate Theory" of pain. The mechanism of its action is not exactly clear, but reports have suggested that it plays the main role in selectively stimulating the large diameter pain fibers in the dorsal aspect of spinal cord. SCS procedure involves a very careful case selection, and current evidence suggests that only a few conditions of chronic refractory neuropathic pain are its established indications. In these patients too, the efficacy rate remains around 50 to $75 \%$. The overall pain relief observed is around $50 \%$ decrease in visual analog scale (VAS) scores. It is a technically simple procedure involving placement of electrodes over the dorsal aspect of spinal cord in the epidural space. The procedure is a staged one in which trial lead electrodes are first implanted and stimulated with an external pulse generator (EPG). If the trial is successful and patient has acceptable pain relief over 1 week of stimulation at various

\section{Keywords}

- spinal cord stimulator

- lead electrodes

- paddle electrodes

- implantable pulse generator

- trial stimulation

- permanent stimulation settings, the patient undergoes the permanent implantation of electrodes at the same position. The permanent electrodes are then stimulated by an implantable pulse generator (IPG) in the subcutaneous pocket (abdominal or gluteal). Complications are rare and are more related to hardware like lead migration and breakage. Since it is does not damage the cord per se, its acceptance as a procedure for pain is known quite well in the Western world. Its availability and cost of implants is the major hurdle in its use in a developing nation like India. Here, we present a technical note and our experience of two cases of thoracic spinal cord stimulation for chronic neuropathic pain at our institution.
\end{abstract}

\section{Introduction}

Spinal cord stimulation (SCS) was developed by Shealy in $1967.1^{1,2}$ It was merely 2 years after the proposal of the "Gate Theory" of pain transmission by Melzack and Wall in $1965 .{ }^{3}$ Since then, it has increasingly been used in treatment of chronic neuropathic pain conditions. The use of electric current for electrical stimulation and its use in pain relief has been known for centuries, but it came to be reasonably recognized only after the explanation by published online

January 12, 2021
DOI https://doi.org/

$10.1055 / \mathrm{s}-0040-1716934$ ISSN 2277-954X. (c) 2021. Neurological Surgeons' Society of India.

This is an open access article published by Thieme under the terms of the Creative Commons Attribution-NonDerivative-NonCommercial-License, permitting copying and reproduction so long as the original work is given appropriate credit. Contents may not be used for commercial purposes, or adapted, remixed, transformed or built upon. (https://creativecommons.org/licenses/by-nc-nd/4.0/)

Thieme Medical and Scientific Publishers Pvt. Ltd. A-12, 2nd Floor, Sector 2, Noida-201301 UP, India 
the Gate theory. It is believed that stimulation of small diameter sensory fibers opens the "gates" and the large diameter fibers close them. Since the large diameter fibers have a lower threshold for depolarization, they may be recruited selectively by an externally applied electrical field to a peripheral nerve. They may be more selectively activated by electrical stimulation of the dorsal aspect of the spinal cord as the large-diameter sensory fibers within peripheral nerves are segregated into the dorsal columns. The primary electrical effect of SCS has been assumed to be mediated by the dorsal columns. SCS is also effective at treating hyperalgesia, which is primarily signaled by large fibers. This indicates that relief of pain by electrical stimulation is due to frequency-related conduction block, acting at primary afferent branch points where dorsal column fibers and dorsal horn collaterals diverge. ${ }^{4}$ SCS, however, has not been found to be really effective in other pain conditions like the pain of acute injury. ${ }^{5}$ Therefore, this gate theory alone fails to explain its ineffectiveness. This suggests that other mechanisms involving interneurons in the dorsal horn or involving descending fibers or sympathetic mechanisms may exist. ${ }^{6}$ Studies have also demonstrated the role of neurotransmitters in patients with SCS. It has been shown that SCS decreases the release of excitatory neurotransmitters like glutamate and aspartate, and increases release of inhibitory ones such as gamma-amino butyric acid (GABA) and substance P (SP). ${ }^{6}$ Having said that, our understanding of the mechanisms underlying SCS is limited.

\section{Indications}

SCS is currently indicated in a variety of chronic neuropathic pain syndromes. In 2014, Nagel and Lempka described the common indications with varying grades of evidence. ${ }^{7} \mathrm{~A}$ few of the specific ones are as follows:

- Failed back surgery syndrome (FBSS): This is the most common indication for SCS. It was supported by the prospective randomized controlled multicenter trial of the effectiveness of SCS (PROCESS trial) in $2005 .^{8}$
- Chronic regional pain syndrome (CRPS) (Type I and II): Also known as reflex sympathetic dystrophy, the condition is of unclear pathophysiology and has very limited treatment options. Kemler et al in their randomized control trial (RCT) have found significantly better relief of symptoms in SCS patients than those with conservative management. ${ }^{9}$

(Please refer to - Table 1 for the current evidence and results pertaining to SCS in chronic neuropathic pain syndromes.)

Other less common ones are as follows:

- Postherpetic neuralgia.

- Pain due to peripheral nerve injury.

- Intercostal neuralgia.

- Phantom limb pain syndrome (PLPS).

- Ischemic pain syndromes due to peripheral vascular disease and intractable angina.

\section{Contraindications $^{7}$}

- Coagulopathy.

- Spinal stenosis.

- Infection.

- Psychiatric disorders-major depression and psychosis.

- Substance abuse.

\section{Role of Psychological Screening}

Screening of patients is one of the practically important issues in SCS. Not all patients with chronic pain respond well to SCS. It may be challenging to distinguish neuropathic pain from other causes of pain. Many clinicians have therefore incorporated psychological screening in their protocols for SCS workup. It helps in identifying patients with major psychiatric morbidity (major depression, psychosis, or substance abuse). It has been reported that psychological screening has some predictive value in selecting patients who would benefit from SCS. ${ }^{10}$ Family support and psychological profile of the patient are very important as the patient and his or her family are active participants in patient care. ${ }^{11}$ However, there is also data to suggest that the predictive value of such psychological testing might be low. ${ }^{12}$

Table 1 Current evidence and results for SCS in chronic neuropathic pain syndromes

\begin{tabular}{|c|c|c|c|c|}
\hline Indication & Study/evidence & Year & Type of evidence & Remarks-main inference \\
\hline \multirow[t]{4}{*}{ FBSS } & PROCESS trial ${ }^{8}$ & 2005 & RCT & Level I evidence for efficacy of SCS \\
\hline & Taylor et al ${ }^{16}$ & 2014 & $\begin{array}{l}\text { Systematic review and } \\
\text { meta-analysis }\end{array}$ & Long-term pain remission with SCS \\
\hline & Grider et al ${ }^{17}$ & 2016 & Systematic review & Level I-II evidence for low-frequency SCS \\
\hline & Cameron $^{18}$ & 2004 & Literature review & Overall success rate of $62 \%$ \\
\hline \multirow[t]{3}{*}{$\begin{array}{l}\text { CRPS } \\
\text { (type I and II) }\end{array}$} & Kemler et al ${ }^{19}$ & 2000 & $\begin{array}{l}\text { RCT } \\
\text { (SCS + physical therapy vs } \\
\text { physical therapy alone) }\end{array}$ & $\begin{array}{l}\text { VAS score decreased by } 3.6 \text { points but } \\
\text { increased in the control group }(0.2) \\
(p<0.001)\end{array}$ \\
\hline & ACCURATE trial ${ }^{30}$ & 2017 & $\begin{array}{l}\text { RCT } \\
\text { (SCS vs. DRG) }\end{array}$ & $\begin{array}{l}\text { Both are good but DRG has higher } \\
\text { statistical significance }\end{array}$ \\
\hline & Harrison et al ${ }^{31}$ & 2017 & Literature review & DRG is safe and effective \\
\hline
\end{tabular}

Abbreviations: DRG, dorsal root ganglion; RCT, randomized control trial; SCS, spinal cord stimulation; VAS, visual analog scale. 


\section{Technical Details}

\section{The Device}

The device consists of the following three components: the stimulator electrodes, the generator, and the programmer.

\section{Stimulator Electrodes}

The electrodes have evolved since they were first introduced. The earlier ones were small with a single contact point and involved a laminectomy for their placement. They had limited access and little intraoperative flexibility. Modern-day electrodes are generally either lead or paddle type, with multiple nonferromagnetic (platinum-iridium) contact points encased in silastic insulating material.

\section{Lead Type}

These are generally inserted in epidural space percutaneously. They were first introduced in the 1970s and initially consisted of a single contact point. Multiple leads were therefore required to achieve bipolar stimulation. By the 1980s, leads with multiple contact points (1-8) in a linear array were available (-Fig. 1). They had improved maneuverability and better stimulation with smaller contact points and lesser intercontact spacing. This resulted in superior targeting of the dorsal columns of the spinal cord..$^{13}$ Further, evolution of the "transverse tripole" concept, which involves a negative terminal flanked transversely by two positive terminals, has significantly optimized the treatment and modulation of pain. This is achieved by placing two leads percutaneously in the epidural space, parallel to each other with contact points staggered.

\section{Paddle Type}

These are paddle-shaped electrodes with two to three columns of disc-shaped electrodes. They are introduced through a laminectomy and placed epidurally. They allow for more focused dispersion of current. They are indicated when there is repeated migration of percutaneous lead type electrodes or there is a technical difficulty in placing percutaneous lead electrodes such as epidural adhesions. They may also be used when there is disease at the level planned for stimulation (hypertrophic ligaments flava). As per the clinical data published in 2005, according to a RCT, ${ }^{14}$ the lead type electrodes

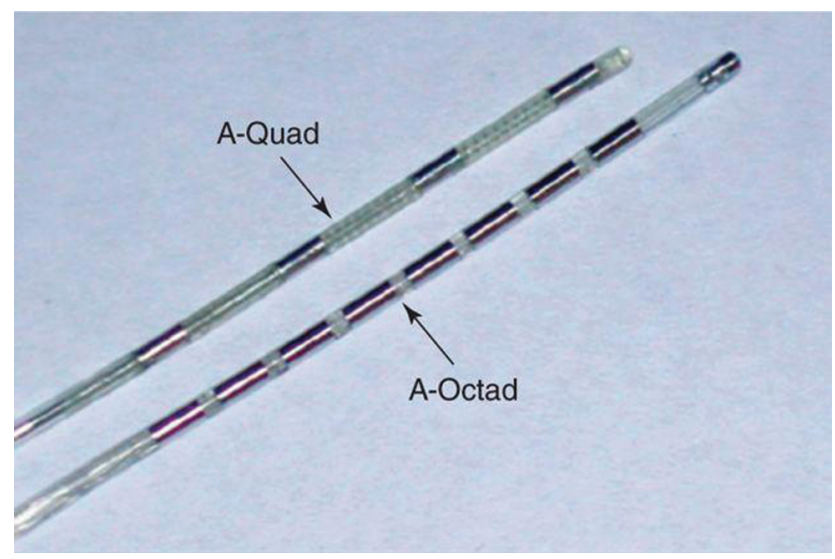

Fig. 1 Lead electrode. had a better coverage of pain and improved low back coverage as compared with paddle type electrodes. However, the lead electrode produces a spherical electrical field and the part in contact with the dural surface is only effective, but paddle electrode's field apposes the spinal cord only and is therefore more effective and requires lesser electrical power. Many centers are increasingly using lead type electrodes for trial version of SCS and paddle leads for permanent stimulation (-Fig. 2).

\section{Generators}

To energize the stimulator electrodes, a generator is required to deliver the current at desired frequency, amplitude, and pulse width. The initial generators were radio-frequency transmitters applied externally with no implanted battery. To wear them continuously was a major disadvantage. By the 1980s, implantable pulse generators (IPG) became available. These are generally implanted either in the lower quadrant of abdominal wall or in the buttocks, depending on patients' choice. They are more cosmetic and convenient. They are available with both permanently charged and rechargeable battery configurations (-Fig. $\mathbf{3}$ ).

\section{Patient Programmers}

Programmer is a device used by the users to modify the settings of stimulation to optimize their pain management. They have become more sophisticated and user-friendly over

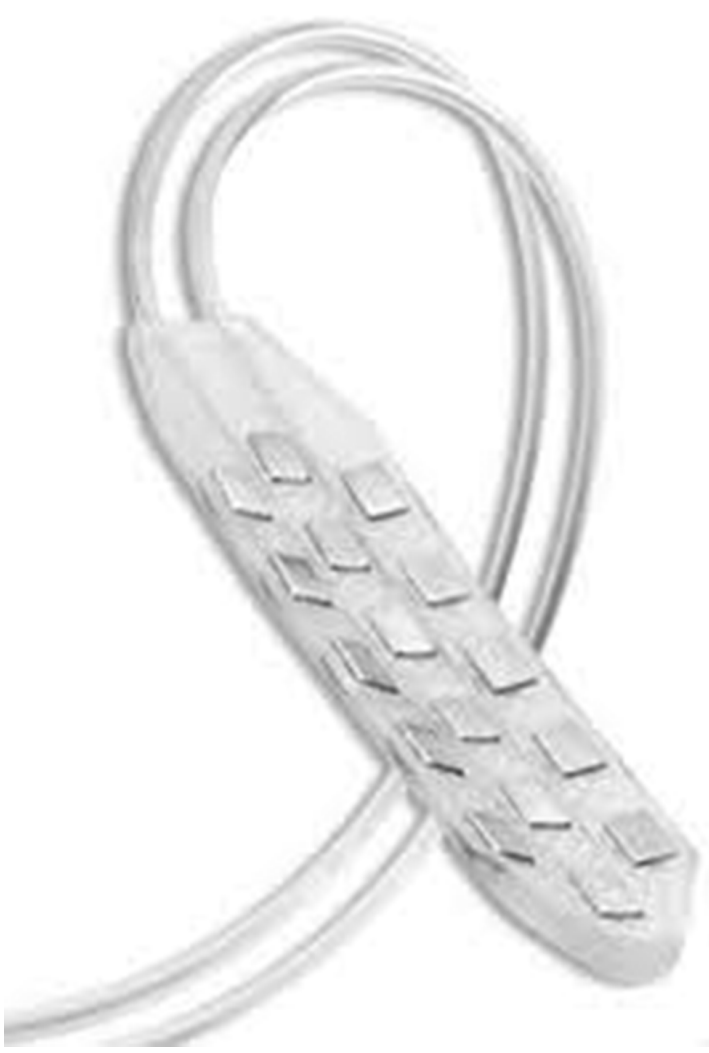

Fig. 2 Paddle electrode. 
the years ( $\boldsymbol{- \text { Fig. }}$ 4). The patients can now self-modulate their settings of frequency, amplitude, pulse width, and contact polarities. With increased advent of more advanced technology, programming and fine tuning of neuromodulation can now be done for the patient at the comfort of his home by the clinician from his/her office.

\section{Preoperative Workup and Patient Education}

The preoperative planning for spinal cord stimulation requires a thorough workup. It involves a clinical evaluation, generally on OPD basis wherein the history of clinical symptoms and the refractoriness of pain is reevaluated. A thorough physical examination and charting of dermatomal distribution of pain is must. A pain scoring protocol is also mandatory. Investigational correlation with appropriate imaging studies to rule out more organic causes is collaborative. The confirmation of diagnosis with a review recommendation by pain physician is preferred although not mandatory. A comprehensive understanding of the algorithm of workup has been highlighted by Dones et al. ${ }^{15} \mathrm{~A}$ schematic representation is described in the flowchart above. An elaborate preanesthetic checkup is required. The importance of a preoperative psychological evaluation cannot be ignored. Finally, the procedure and the outcomes have to be discussed in detail with the patient and his or her family, as this procedure involves their significant involvement in comprehensive patient care. It has to be explained to the patient that the procedure is carried out in two stages. First is the trial lead placement under local anesthesia at the desired level. The patient would be awake and would be required to cooperate intraoperatively as to ascertain whether all symptomatic dermatomes have been covered on table. The patient also needs to understand that the lead ends would come out externally and will be connected to the external pulse generator (EPG). They also must understand what to expect at trial stimulation intraoperatively. It is mild tingling in the affected area or limb. It is actually patient-guided level of stimulation intraoperatively. The trial of stimulation with various settings (pulse, frequency, and voltage) and modes (tonic, burst, or high-frequency modes) commences in the next 1 week and titrated according to the level of pain relief. The final settings are optimized and recorded. In the second stage, if the trial stage is successful, permanent lead placement with IPG is done under general anesthesia. If otherwise, removal of trial electrodes becomes the only option.

\section{Surgical Procedure steps}

The first step is placement of trial leads. The procedure is done while the patient is awake. The patient is put in prone position with all pressure points padded by soft silicon bolsters and gel pads. Some short-acting sedation is given to calm the patient and remove the apprehension of surgery. It must be only a light degree of sedation, as the patient has to cooperate throughout the process of trial leads placement and on table stimulation (-Fig. $\mathbf{5}$ ).

It is advisable to mark the site of entry of electrode placement by a permanent skin marker under X-ray guidance (usually L1-L2 for thoracic SCS for FBSS). It is also preferred that a metallic marker be placed on the skin at the proposed superior limit of lead placement (usually the lower border of D8 vertebra; - Fig. 6A). Usual antiseptic preparation and draping is done. Local anesthetic infiltration is done and a small 2 to 3 inch midline incision is made. We usually make a subcutaneous pocket by undermining skin, so as to place the anchors and connectors. The fascia is exposed and midline identified.
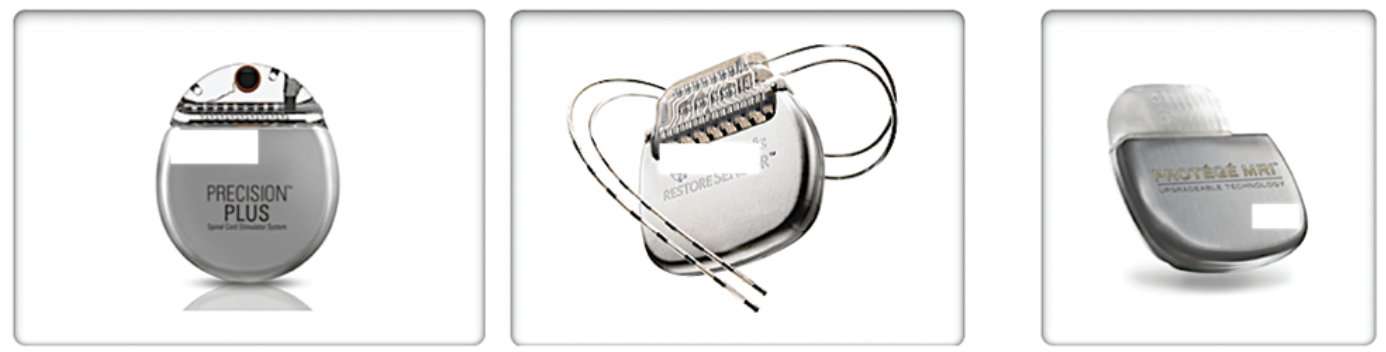

Fig. 3 Types of implantable pulse generators (IPG).
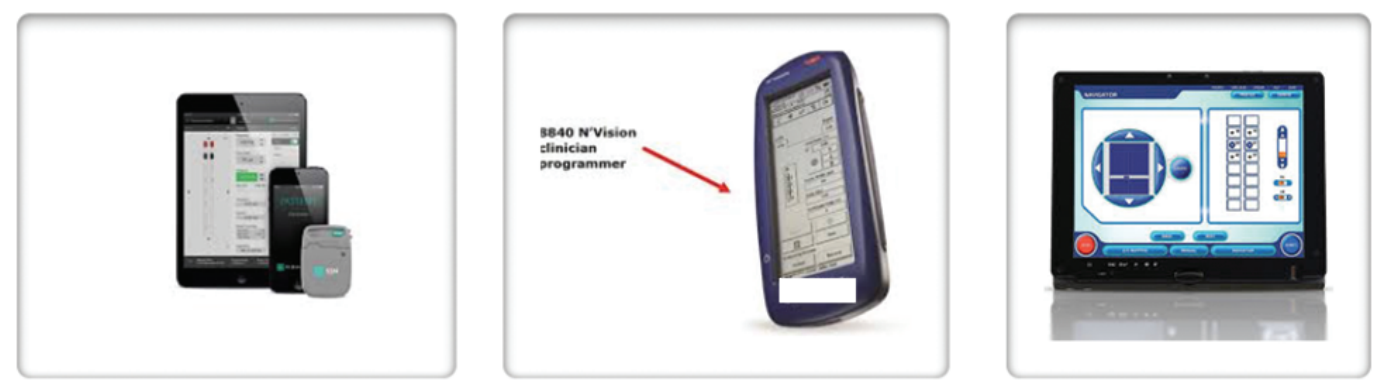

Fig. 4 Patient programmers. 
For lead electrode placement, localize under the spinous process. In the area just paramedian to the spinous process, puncture with a Tuohy needle and advance obliquely and superiorly (under fluoroscopic guidance) almost at an angle of 30 to 45 degrees from fascial plane (-Fig. 6B). When the lower border of the superior lamina is hit, advance a tip of the needle a little deep to get into epidural space. It is indicated by a loss of resistance (LOR). Confirm with a LOR syringe and the image on fluoroscopy. Dural puncture is indicated by egress of cerebrospinal fluid (CSF) and warrants reinsertion. Remove the stylet and put a dilator to set the direction for the passage of the electrode just lateral to the midline ( - Fig. $6 \mathbf{6}$ ). Slowly remove the dilator from the needle, not disturbing its position in the epidural space. Gently thread the lead electrode (with its stylet) epidurally through the Tuohy needle and advance electrode vertically up in the spinous line (midline) under X-ray fluoroscopic guidance ( Fig. 6D, E). The upper limit of advancement is decided by the metal marker attached to the skin placed previously. For the other side, perform the same technique similarly ( $\mathbf{- F i g . 6 G}$ ). It is typically advised that the contact points of the electrodes on the leads should be "staggered" and one lead should lie at a vertebra 1 level below the proposed upper limit ( - Fig. 6G, H). The "anchors" on the leads are placed and anchored to the fascia. Strain relief loops are given, and the distal ends of the leads are connected to

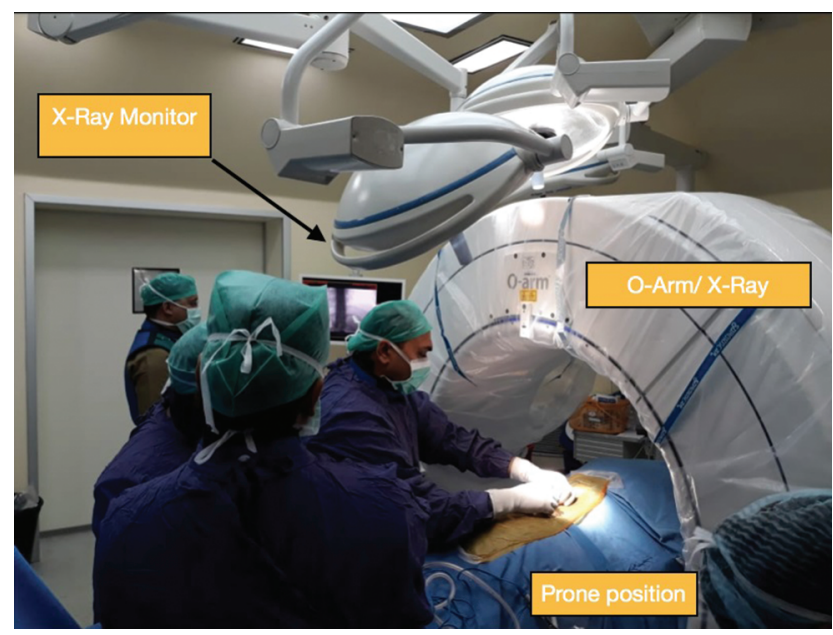

Fig. 5 Patient positioning and operating room setup. the "connectors," which are finally tunneled out from the skin reasonably away $(10-12 \mathrm{~cm})$ from the midline wound ( - Fig. 6F). The midline incision is closed in layers. The position of leads and contact points of electrodes is reconfirmed. (Please refer to - Fig. 6 for a pictographic representation of surgical steps under X-ray fluoroscopic guidance.)

Placement of paddle electrodes the technique is a little different. The midline incision is taken at the level where the contact points need to rest epidurally. Localize again on X-ray and mark the area. After routine skin preparation and draping, the midline incision is taken and deepened. The spinous process is exposed and attached muscles are retracted away. A small laminectomy is performed and adequate space is created for the paddle lead to slide epidurally. Often a special dissector and a specially designed bayonet is used to slide the paddle electrode on the epidural space. Again, we anchor the electrode in position with "anchors" and connect the distal ends to the connectors. Tunnel the connectors through separate punctures on the skin approximately 10 to $12 \mathrm{~cm}$ away from the primary incision. The midline incision is closed in layers.

The ends of connectors are attached to an EPG and impedances are checked. On table stimulation is done to check the tingling or numbness in the dermatomes involved. The patient should be able appreciate a "light rumble of fingers"-like sensation on the involved dermatome. The technician should then report the dermatomes as "covered" on the intraoperative stimulation. Once all involved dermatomes are covered on the intraoperative trial of stimulation, the connectors are anchored to the skin with sutures. The external cables of the connector (sometimes along with the EPG) are secured to the skin with adhesive tapes. All wounds are dressed and patient is shifted to recovery.

\section{Trial of Stimulation}

The formal trial of stimulation begins immediately and then continues for the next 1 week by the trained company technician in different modes and settings, which is done in consultation with the neurosurgeon. The stimulation can be tonic, high-frequency, or burst type, depending on the device and vendor. Conventional SCS is a constant tonic stimulation. Newer and more advanced systems have
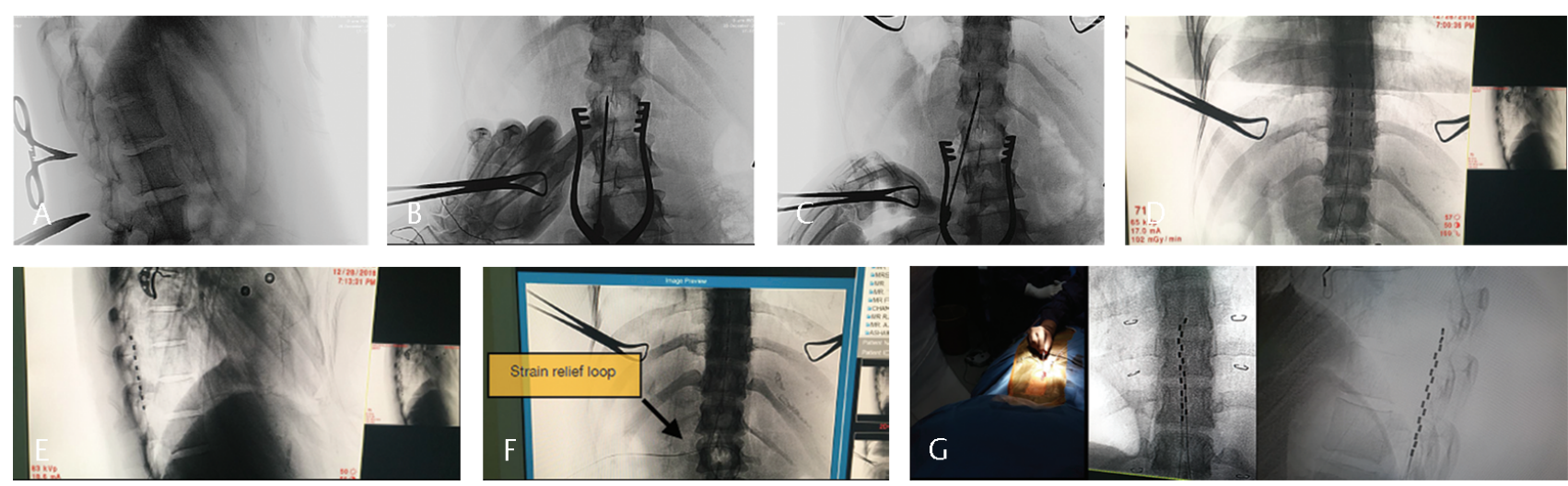

Fig. 6 (A-G) Pictographic representation of surgical steps under X-ray fluoroscopic guidance. 
incorporated high-frequency and burst stimulation

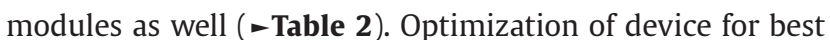
relief of symptoms without side effects is done and settings are recorded.

A pain relief scoring is done on available pain scoring modules and a log chart is prepared for modulation. Subjectively, the patient experiences mild tingling in the stimulated dermatome. No numbness or loss of sensation is expected and should be treated as a side effect.

A trial is labeled "successful" if there is pain relief of at least $50 \%$ in at least half of the dermatomes covered. If there any major side effects like loss of sensation or there is minimal relief of sensation, the trial is labeled "unsuccessful." The patient and the family is then counselled that the trials have not worked for the patient and should be removed. On the success of trial stimulation, the second step of the procedure is planned.

\section{Placement of Permanent Electrodes and IPG}

Permanent electrodes are generally placed under general anesthesia with the patient either prone or lateral (depending on the area for proposed IPG, gluteal or abdominal pocket). Currently, permanent electrode placement is practiced in two ways. Some clinicians, especially "pain physicians," remove the trial assembly completely and reimplant fresh electrodes. They generally use the trial electrode (without connectors) for trial stimulation only. However, others do not remove the stimulating electrodes; instead, they disconnect the connectors from the trial leads and cut the exposed connecting cable. The advantage is that the position of contacts that gave the best relief on trial remains same. This translates into a better pain relief, as the physical and modulation settings remain same. Another advantage is the reduction in cost of this already costly procedure. The connectors are then completely removed and the distal ends of the trial leads are tunneled subcutaneously to the pocket for IPG.

The IPG can be placed in a subcutaneous pocket in the lower quadrant of abdomen above the waistline (to avoid seat belt impression) or in the buttocks, as per the patient's convenience. The strain relief loops (on the extra length of lead) are given and placed under the IPG, so that they do not interfere with stimulation and modulation from external patient programmer. All wounds are irrigated with antiseptic solution and closed in layers. No drainage is required. Salient features of trial and permanent stages are summarized in - Table 3.

\section{Neuromodulation after Permanent SCS}

The patient generally stays in the hospital for a couple of days. Neuromodulation is done daily to take optimum effect. A company technician in consultation with the primary

Table 2 Stimulation parameters in various modes of stimulation in SCS for neuropathic pain

\begin{tabular}{|l|l|l|l|}
\hline Type of Stimulation & Frequency & Pulse width & Amplitude (current) \\
\hline Tonic $^{25,26}$ & $40-80 \mathrm{~Hz}$ & $200-450 \mu \mathrm{s}$ & $0-25.5 \mathrm{~mA}(\mathrm{max} 12 \mathrm{~V})$ \\
\hline Burst $^{27}$ & $\begin{array}{l}\text { Five high frequency stimuli at } \\
500 \mathrm{~Hz}, 40 \text { times per second }\end{array}$ & $\begin{array}{l}50-1000 \mu \mathrm{s} \text { with interspike } \\
\text { interval of } 1000 \mu \mathrm{s} \text { over a } \\
\text { constant tonic firing }\end{array}$ & $0-12.75 \mathrm{~mA}$ \\
\hline High frequency & 25,28 & $30 \mu \mathrm{s}$ & $3 \mathrm{~V}^{29}$ \\
\hline
\end{tabular}

Abbreviation: SCS, spinal cord stimulation.

Table 3 Salient points

\section{Trial and permanent stages in SCS-salient points}

Trial

- Lead electrodes placement at desired level with connectors under LA (awake)

- Connect the distal ends of connectors to EPG.

- "Trial" of stimulation at various settings for next 1 week (with the help of company technician).

- Neuromodulation according to subjective relief of the patient and the areas "covered."

- Assess at 1 week for "success" or "failure" of trial stage.

- If the trial SCS was successful, go ahead with permanent SCS.

- If the trial SCS failed, remove implants under LA.

Permanent

- Reimplantation of lead electrodes at the same position as trial (some centers just use the same electrodes and just remove the connectors, while others use new set of lead (or sometimes paddle) electrodes.

- Distal ends of electrodes tunneled to a subcutaneous IPG pocket (subcostal or gluteal)

- Stimulation parameters set the same as the ones successful at trial.

- Fine tuning of neuromodulation done according to patient to further control pain.

- Discharge after a couple of days with dressing change.

Abbreviations: EPG, external pulse generator; IPG, implantable pulse generator; SCS, spinal cord stimulation. 
surgeon modulates the settings for the best subjective pain relief. Pain scores are calculated and recorded. The patient is called for follow-up 1 week later and then monthly for next 3 months. It is advisable that patient is further followed-up at 6 months, 1 year, and 2 years after the procedure.

\section{Results}

SCS success rates are generally in the range of approximately 50 to $75 \%$ when the case selection is appropriate and the team is experienced. Despite careful selection, quite a few patients do not benefit from SCS. There is an average decrease in pain of $50 \%$ among the responders. The reason for such variable response from SCS is still unclear. The following table is a literature review of the responsiveness to SCS in various studies (-Table 1).

There is a Level I evidence for SCS in FBSS (see PROCESS trial). ${ }^{8}$ Taylor et al found long-term remission of pain after SCS in a systematic review and meta-analysis. ${ }^{16}$ It has been further supported by studies done by Grider et al and Cameron et al. ${ }^{17,18}$

Complex regional pain syndrome (CRPS) (both type I and II) also responds quite well to SCS. In fact, Kemler et al published a randomized trial in 2000 and suggested that SCS with physical therapy resulted in a decrease of VAS scores by 3.6 in CRPS patients when compared with physical therapy alone (VAS score actually increased by 0.2 in this arm) ${ }^{19}$

\section{Complications}

Spinal cord stimulator placement is generally a well-tolerated procedure. Bleeding, infection (2.4\% to $18.6 \%$ ), and wound dehiscence are very rare. ${ }^{20,21}$ More important are issues like electrode migration and electrode breakage (24-50\%). ${ }^{22-24}$ Migration of electrodes is generally avoided by putting strain relief loops and abdominal placement of IPG. They are more common when the IPG is placed in the buttock area due to direct transmission of stretch due to spinal movements. There can be lead or electrode breakage (usually near the anchors). It is best avoided by placing the anchors about halfway inside the fascia and then securing it with sutures. The traction over the lead is thus avoided near the anchors and chances of breakage are therefore lesser.

Patients may also complain of loss of sensation in the stimulated dermatome. The settings can be modulated but if the issue persists, the stimulator may warrant removal in a rare circumstance. The patient may also experience increased tingling, affecting normal activity. Modulation of settings of stimulation generally takes care of this problem.

\section{Precautions and Advisories}

- Uncontrolled heating of the electrode may be caused by sources of electrical and magnetic energy.

- Modern leads using titanium are safer and also MRI compatible.

- Avoid use of monopolar cautery.

- Diathermy during physiotherapy should be avoided.

- Avoid proximity to these antitheft devices.

\section{Our Cases}

\section{Case 1}

Our first case is a young woman of 25 years of age, a business executive by occupation. She was operated for a lumbar lipomyelomeningocele in 2010. She had a trivial fall approximately 3 weeks after the surgery. Initially, she started having pain in the back of thigh and left gluteal region. This was managed conservatively and it resolved completely after about a month. The wound also healed well. She remained asymptomatic till about mid 2016 when she started experiencing low backache. She presented with worsening low backache with continuous radiating pain over bilateral lower limbs. She also developed numbness in the left gluteal region. Clinically, she had pain in L1-S1 dermatomes bilaterally (VAS score of 9/10). There was some loss of sensation in left S1 dermatome. However, there was no weakness and the straight leg raising (SLR) tests were bilaterally positive. An MRI of the LS spine suggested residual intradural lipomatous lesion at L2-4 level with low-lying conus. Since the pain was the main complaint and the patient did want any aggressive surgical management, a spinal cord stimulator was offered as a therapy to alleviate pain. Repeated counseling sessions of the patient and the family were done, and course and expected outcome were explained.

She underwent the SCS trial on December 2018. Under local anesthesia and mild sedation, with the patient awake, a midline incision was done and a subcutaneous pocket was created. We implanted two lead electrodes (eight contact points) and placed them from T8 lower border to T10 lower border in a fashion that the contact points were "staggered," so as to achieve the "triple tripole effect." The lead electrodes were connected to connector leads which were brought out percutaneously. The leads were anchored as described and the wound closed. The trial of stimulation was given "on table," and she herself felt that all areas in question were covered.

For the next 1 week, a daily modulation of the settings of stimulation were done. She performed very well with adequate pain relief (VAS1-2/10) in almost all affected dermatomal areas. Trial was considered as "successful," as the pain relief was greater than $70 \%$. She was offered the permanent SCS.

Permanent SCS was done under general anesthesia with patient being in lateral position. We reopened the midline wound and disconnected the connectors from the distal end of the "trial" lead electrodes. The connectors were then removed after cutting them near the skin surface. The ends of the implanted lead were then tunneled subcutaneously to an abdominal pocket and connected to the IPG. The IPG battery was rechargeable type. All wounds were closed.

The neuromodulation began the following day, and she experienced very good pain relief which was comparable to the one after trial stimulation period (VAS: 1-2/10). This remained for next couple of days after which she was discharged and was kept on follow-up. On her three follow-ups in over the course of 1 year, we observed good 
control of her pain (VAS: 3/10); however, the numbness in left gluteal region remained. We changed the settings marginally on each follow-up, and the overall response of the patient continues to be encouraging till our last follow-up in February 2020.

\section{Case 2}

Our second case was a 75-year-old retired Chief of Police from Malawi. He was diabetic since 1994. His symptoms started in 1994 when he started experiencing back pain. Gradually, his pain started radiating to his both lower limbs and also in his anal area. By 1995, he started having numbness in his both hands and feet. In 1998, he was sent to South Africa (SA) for evaluation. He was treated conservatively there. After returning, he resumed his office but started experiencing progressive difficulty in sitting, typing records on the computer, and performing his other professional obligations. He started going on multiple work leaves due to pain. In 2006, he was sent to South Africa again and underwent multiple evaluations by several teams. They finally diagnosed him to have $\mathrm{C} 1-\mathrm{C} 2$ fracture and offered him fixation with a 50\%chance of improvement of his symptoms. He was operated in SA in 2006 and was sent back to Malawi on a physiotherapy and regular physical exercise schedule. He had to report back for follow-up in April 2007, but could only manage to be there by December 2007. By then, he had extreme neck and back pain with restriction of movements of neck. He was again evaluated in SA and was found to have loosened implants. He was therefore operated again in early 2008 and all implants were replaced by new ones. He was again sent back to his native country, but he continued to have neck and back pain with intermittent severe exacerbations. His back pain started worsening in the last couple of years, which has restricted him from walking. He also felt progressive numbness in his hands and feet.

He presented to us with the above history and remained confined to bed most of the times throughout the day. He also had agonizing pain in the perianal area which exacerbated on sitting. He could barely turn in bed because of the back pain. He sometimes got on a wheelchair/motorized scooter for mobility when assisted by a couple of people. He had difficulty moving both lower limbs because of pain. He had been catheterized because of his immobility and failure to control his urination. He also complained of erectile dysfunction for the past several years.

On examination, he was found to have a depressed look with dependence on his wife for most issues, although he was very clear on his history and what he was suffering. He was obese and had some swelling in bilateral hands and feet. He had restriction of neck movements with almost $4 / 5$ power in upper limbs. There was no appreciable sensory loss in upper limbs, but he kept complaining that the tips of fingers were numb. His lower limb examination revealed a power of $3 / 5$ at bilateral hips and knees and $2 / 5$ at ankle (painful). He experienced pain in the back, and it was difficult for him to even turn him to examine his back. Anal examination did not reveal any fissure sinus or hemorrhoids but extreme tenderness was felt over the coccygeal tip. It was suggestive of coccydynia.

His dermatomal chart for pain distribution is mentioned below.

His pain score was as follows:

VAS: 9/10 at L4-S3 dermatomes bilaterally.

VAS: 8/10 at D9-L3 dermatomes bilaterally.

VAS: 6/10 at C3-T1 dermatomes bilaterally.

SCS was therefore offered as a procedure to alleviate pain only. It was communicated that it is unlikely to help in his spastic weakness or erectile dysfunction, and that he would require regular and long-term physiotherapy for his weakness.

The patient and his wife were explained the trial and permanent protocols of stimulation, and once they were ready, the trial procedure was carried out. He responded well to the trial stimulation, initially, for a couple of days. When we modulated him on a different setting, his response got reduced. After many permutations of settings, a reasonable setting was reached and good pain relief was observed. He could sit up, walk, and mobilize. After a week of trial stimulation, it was converted to a permanent procedure and IPG was placed in abdominal pocket. After permanent implant, he was so happy that he actually danced to his favorite song. Multiple steins were offered to relieve the different types of pain he experienced. His anal pain had completely disappeared. His VAS scores on the day of discharge were as follows:

VAS: 4/10 at L4-S3 dermatomes bilaterally.

VAS: 4/10 at D9-L3 dermatomes bilaterally.

VAS: 3/10 at C3-T1 dermatomes bilaterally.

This suggested that the SCS was working well for him with greater than $50 \%$ pain relief. He has been doing well till his last follow-up in December 2019.

\section{Conclusion}

SCS is an established procedure and is gaining some ground in developing country like ours. Its use is limited by availability, cost, and the number of centers in India doing it. Nevertheless, it is a very simple and effective procedure for chronic neuropathic pain syndromes. It involves a dedicated team effort to achieve optimal results. The coordination between the neurosurgeon, neuroanesthetist, pain physician, clinical psychologist, and company technician is a prerequisite. It is imperative to reiterate that patient selection, explanation of the procedure and the expected outcome, and cooperation of the patient are very important variables to the success of the SCS procedure.

Conflict of Interest

None declared. 


\section{References}

1 Shealy CN, Mortimer JT, Reswick JB. Electrical inhibition of pain by stimulation of the dorsal columns: preliminary clinical report. Anesth Analg 1967;46(4):489-491

2 Shealy CN, Taslitz N, Mortimer JT, Becker DP. Electrical inhibition of pain: experimental evaluation. Anesth Analg 1967; 46(3):299-305

3 Melzack R, Wall PD. Pain mechanisms: a new theory. Science 1965;150(3699):971-979

4 Schechtmann G, Song Z, Ultenius C, Meyerson BA, Linderoth B. Cholinergic mechanisms involved in the pain relieving effect of spinal cord stimulation in a model of neuropathy. Pain 2008;139(1):136-145

5 Kavar B, Rosenfeld JV, Hutchinson A. The efficacy of spinal cord stimulation for chronic pain. J Clin Neurosci 2000;7(5): 409-413

6 Cui JG, O'Connor WT, Ungerstedt U, Linderoth B, Meyerson BA. Spinal cord stimulation attenuates augmented dorsal horn release of excitatory amino acids in mononeuropathy via a GABAergic mechanism. Pain 1997;73(1):87-95

7 Nagel SJ, Lempka SF, Machado AG. Percutaneous spinal cord stimulation for chronic pain: indications and patient selection. Neurosurg Clin N Am 2014;25(4):723-733

8 Kumar K, Taylor RS, Jacques L, et al. Spinal cord stimulation versus conventional medical management for neuropathic pain: a multicentre randomised controlled trial in patients with failed back surgery syndrome. Pain 2007;132(1-2):179-188

9 Kemler MA, de Vet HC, Barendse GA, van den Wildenberg FA, van Kleef M. Effect of spinal cord stimulation for chronic complex regional pain syndrome Type I: five-year final follow-up of patients in a randomized controlled trial. J Neurosurg 2008; 108(2):292-298

10 Doleys DM. Psychological factors in spinal cord stimulation therapy: brief review and discussion. Neurosurg Focus 2006;21(6):E1

11 Kumar K, Hunter G, Demeria D. Spinal cord stimulation in treatment of chronic benign pain: challenges in treatment planning and present status, a 22-year experience. Neurosurgery 2006; 58(3):481-496, discussion 481-496

12 North RB, Kidd DH, Wimberly RL, Edwin D. Prognostic value of psychological testing in patients undergoing spinal cord stimulation: a prospective study. Neurosurgery 1996;39(2):301-310, discussion 310-311

13 Holsheimer J. Computer modelling of spinal cord stimulation and its contribution to therapeutic efficacy. Spinal Cord 1998;36(8):531-540

14 North RB, Kidd DH, Olin JC, Sieracki JM. Spinal cord stimulation electrode design: prospective, randomized, controlled trial comparing percutaneous and laminectomy electrodes-part I: technical outcomes. Neurosurgery 2002;51(2):381-389, discussion 389-390

15 Dones I, Levi V. Spinal cord stimulation for neuropathic pain: current trends and future applications. Brain Sci 2018; $8(8): 138$
16 Taylor RS, Desai MJ, Rigoard P, Taylor RJ. Predictors of pain relief following spinal cord stimulation in chronic back and leg pain and failed back surgery syndrome: a systematic review and meta-regression analysis. Pain Pract 2014;14(6):489-505

17 Grider JS, Manchikanti L, Carayannopoulos A, et al. Effectiveness of spinal cord stimulation in chronic spinal pain: a systematic review. Pain Physician 2016;19(1):E33-E54

18 Cameron T. Safety and efficacy of spinal cord stimulation for the treatment of chronic pain: a 20-year literature review. J Neurosurg 2004;100(3,Suppl Spine):254-267

19 Kemler MA, Barendse GA, van Kleef M, et al. Spinal cord stimulation in patients with chronic reflex sympathetic dystrophy. N Engl J Med 2000;343(9):618-624

20 May MS, Banks C, Thomson SJ. A retrospective, long-term, third-party follow-up of patients considered for spinal cord stimulation. Neuromodulation 2002;5(3):137-144

21 Hoelzer BC, Bendel MA, Deer TR, et al. Spinal cord stimulator implant infection rates and risk factors: a multicenter retrospective study. Neuromodulation 2017;20(6):558-562

22 Levy RM. Device complication and failure management in neuromodulation. Neuromodulation 2013;16(6):495-502

23 Eldabe S, Buchser E, Duarte RV. Complications of spinal cord stimulation and peripheral nerve stimulation techniques: a review of the literature. Pain Med 2016;17(2):325-336

24 Shamji MF, Westwick HJ, Heary RF. Complications related to the use of spinal cord stimulation for managing persistent postoperative neuropathic pain after lumbar spinal surgery. Neurosurg Focus 2015;39(4):E15

25 Shechter R, Yang $\mathrm{F}$, $\mathrm{Xu} \mathrm{Q}$ et al. Conventional and kilohertz-frequency spinal cord stimulation produces intensity- and frequency-dependent inhibition of mechanical hypersensitivity in a rat model of neuropathic pain. Anesthesiology 2013;119(2):422-432

26 Yearwood TL, Hershey B, Bradley K, Lee D. Pulse width programming in spinal cord stimulation: a clinical study. Pain Physician 2010;13(4):321-335

27 De Ridder D, Vanneste S, Plazier M, van der Loo E, Menovsky T. Burst spinal cord stimulation: toward paresthesia-free pain suppression. Neurosurgery 2010; 66(5):986-990

28 De Ridder D, Perera S, Vanneste S. Are $10 \mathrm{kHz}$ stimulation and burst stimulation fundamentally the same? Neuromodulation 2017;20(7):650-653

29 Arle JE, Mei L, Carlson KW, Shils JL. High-frequency stimulation of dorsal column axons: potential underlying mechanism of paresthesia-free neuropathic pain relief. Neuromodulation 2016;19(4):385-397

30 Deer TR, Levy RM, Kramer J, et al. Dorsal root ganglion stimulation yielded higher treatment success rate for complex regional pain syndrome and causalgia at 3 and 12 months: a randomized comparative trial. Pain 2017;158(4):669-681

31 Harrison C, Epton S, Bojanic S, Green AL, FitzGerald JJ. The efficacy and safety of dorsal root ganglion stimulation as a treatment for neuropathic pain: a literature review. Neuromodulation 2018;21(3):225-233 